der Na-K-Verteilung möglich waren $(1,2,3)$, sind beim Mg nicht möglich. Dazu müßte die Konzentration des ionisierten $\mathrm{Mg}$ unter den jeweiligen Bedingungen in der Zelle bekannt sein.

$\mathrm{Da} \mathrm{Mg}$ zahlreiche Enzyme in der Zelle aktiviert, ist zu diskutieren, ob die beobachteten Änderungen des $\mathrm{Mg}$ Gehaltes ursächlich an der Wirkung der verschiedenen Hormone beteiligt sind. Die Änderung des intrazellulären Gesamt-Mg-Bestandes sind zwar gering. Wie diese
Änderungen des Mg-Bestandes die einzelnen Zellfraktionen (wie Mitochondrien, Ribosomen, Cytoplasma usw.) betreffen, ist unbekannt. Wie sich unter hormonellen Einflüssen die Konzentrationen an $\mathrm{Mg}$-Komplexbildnern in diesen Zellorganellen verhalten, ist ebenfalls unsicher. Deshalb lassen sich noch keine Aussagen über die $\mathrm{Mg}$-Ionenkonzentrationen an den Wirkungsorten des $\mathrm{Mg}$ in der Zelle und über eine Beteiligung des $\mathrm{Mg}$ am Zustandekommen von Hormonwirkungen machen.

\title{
Literatur
}

1. Dulce, H.-J., Th. GüNther und E. Schütte, Clin. Chim. A. 3, 423 (1958). - 2. Dulce, H.-J. und Th. GüNther, Arch. Exp. Path. Pharm. 238, 368 (1960). - 3. GüNther, Th., H.-J. Dulce und E. Schürte, Arch. Exp. Path. Pharm. 239, 283 (1960). - 4. GüNThER, Th., Habilitationsschrift, Berlin (1965). - 5. WeICHSELbaum, T. E., Amer. J. Chem. Path. 10, 49 (1956). - 6. Orange, M. und H. C. RheIN, J. biol. Chem. 189, 379 (1951). - 7. Willis, W. J. und F. W. Sunderaran, J. biol. Chem. 197, 343 (1952). 8. Fisher, R. A., Statistische Methoden für die Wissenschaft, Oliver and Boyd (1956). - 9. Lr, C. H., H. PAPkofF und C. W. Jordan, Proc. Soc. exp. Biol. Med. 100, 44 (1959). - 10. Hanna, S., L. McINTyre, M. T. HARrison und R. Fraser, Btit.Med. J.II, 12 (1961). - 11. NoAll, M. W., T. R. Riggs, L. M. WALKeR und H. N. Christensen, Science 126, 1002 (1957). - 12. Henderson, M. J., H. E. Morgan und C. R. Park, J. biol. Chem. 236, 2157 (1961). - 13. Da Vanzo, J. P., H. C. CRossfield und W. W. Swingle, Endocrin. 63, 825 (1958). - 14. Conway, E. J. und D. Hingerty, Biochem. J. 40, 561 (1946). - 15. Harrop, G. A., L. J. Soffer, R. ElLSWORTh und J. H. Trescher, J. exp. Med. 58, 17 (1933). - 16. ZwEMER, R. L. und R. C. SulLIVAN, Endocrin. 18, 97 (1934). - 17. MAder, J. und L. T. Iseri, Amer. J. Med. 19,
976 (1955). - 18. HrNGerTy, D., Biochem. J. 66, 429 (1957). 19. HanNa, S. und I. McIntyre, Lancet. II, 348 (1960). - 20. Ross, D. B. und A. D. Care, Biochem. J. 82, 21 (1962). - 21. Arkawa, J. K., E. L. Rhoades, D. R. Harms und J. Z. Reardon, Amer. J. Physiol. 197, 99 (1959). - 22. Kerppola, W., Endocrin. 67, 252 (1960). - 23. Strrckland, E. H., Arch. Biochem. Biophys. 100, 110 (1963). - 24. Cope, L. C. und N. WolfF, Biochem. J. 36, 413 (1942). - 25. KleEMAN, C. R., F. H. Epstein, D. KAY und E. Taborskx, J. Clin. Endocr. Metabol. 18, 1111 (1958). 26. Bisser, G. W., Amer. J. Med. Sci. 210, 195 (1945) zit. n. Simon, K. H., „Magnesium“ Stuttgart (1964) S. 29. - 27. Arkawa, J. K., Proc. Soc. exp. Biol. Med. 104, 594 (1960). — 28. Martius, C. und B. Hess, Biochem. Z. 326, 191 (1955): - 29. BarNes, B. A., S. M. Krane und O. Cope, J. Clin. Endocr. Metabol. 17, 1407 (1957). - 30. Greenberg, D. M., J. biol. Chem. 98, 765 (1932). 31. MCINTXRe, I., Nature 198, 1058 (1963). - 32. BuLger, H. A. und Mitarbeiter, J. Clin. Invest. 12, 1135 (1932). - 33. BARNES, B. A. und Mitarbeiter, J. Clin. Endocr. Metabol. 17, 1407 (1957). 34. von Nrda, S., zit. n. SrmọN, K. H., „Magnesium“ Stuttgart (1964) S. 20. - 35. WalaAs, O., Acta Physiol. Scand. 21, 27 (1950). - 36. Best, F. A. und V. R. Pickles, J. Endocrin. 32, 121 (1965).

Priv.-Doz. Dr. Th. Günther 1 Berlin 33, Arnimallee 22

\section{Zur Bestimmung des Kreatinins bei Ketonämie}

\author{
Von R. KattermanN \\ Aus der Medizinischen Universitätsklinik Göttingen (Direktor: Prof. Dr. W. Creutzfeldt)
}

(Eingegangen am 2. Juni 1966)

Da die Ketonkörper Aceton und besonders Acetacetat eine positive JAFFE-Reaktion geben, kann bei Ketonämie ein erhöhter Serumkteatiningehalt vorgetäuscht werden. Die Absorptionsspektren der mit Aceton, Acetacetat und Kreatinin gebildeten Farbstoffe sind praktisch identisch mit einem Maximum bei 490-500 $\mathrm{m} \mu$. Dagegen verhalten sich die molaren Extinktionskoeffizienten bei $546 \mathrm{~m} \mu$ in der Farbreaktion nach POPPER wie $1: 10: 150$, nach BoNSNEs wie $1: 20: 1000$. Kurzes Erhitzen eines Acetacetat-haltigen Serumüberstandes beseitigte den Ketonkörper-Störeffekt vollständig; damit ist es möglich, bei Patienten mit Ketoacidose zuverlässige Kreatininwerte zu errhalten.

Ketone bodies, especially acetoacetate, give a positive JAFFE reaction; in ketonaemia they may cause the determination of false "creatinine" values in the blood. The absorption spectra of the colours produced with acetone, acetoacetate and creatinine are practically identical, with maxima at $490-500 \mathrm{~m} \mu$. The molar extinction coefficients of the colour at $546 \mathrm{~m} \mu$ however, are $1: 10: 150$ according to Popper, and $1: 20: 1000$ according to BonsNes. The interference by ketone bodies in acetoacetate-containing serum supernatant is completely removed by brief heating; thus reliable creatinine values may be obtained for patients with ketoacidosis.

In der klinischen Routinediagnostik werden nicht selten Kreatininbestimmungen im Serum von Patienten veranlaßt, bei denen eine Erhöhung der Ketonkörper im Blut (z. B. Praecoma oder Coma diabeticum, Schwangerschaftsketonämie, ketonämisches Erbrechen bei Kindern) vorliegt. $\mathrm{Da}$ Acetacetat und Aceton mit alkalischem Pikrat eine Rotfärbung ergeben, resultieren in solchen
Fällẹn erhöhte „Kreatinin“-Werte, die evtl. Anlaß zu falschen differentialdiagnostischen Überlegungen sein können. $\mathrm{Da}$ in der Literatur keine näheren Angaben über diesen störenden Einfluß der Ketonkörper auf die Bestimmung des Kreatinins im Serum bzw. Urin vorlagen, andererseits in den letzten Jahren spezifische, enzymatische Bestimmungsmethoden ausgearbeitet wor- 
den waren, lag es nahe, das Verhalten der Ketonkörper in der Reaktion mit alkalischem Pikrat nach JAfre (1) eingehender zu untersuchen.

\section{Methodik}

Für die Modellversuche wurden Seren gepoolt, deren Kreatiningehalt bei der Messung im Auto-Analyzer nicht über $1,1 \mathrm{mg} \%$ lag. Nach Zusatz entsprechender Mengen Acetacetat wurden solche Seren - ebenso wie wäßrige Standardlösungen von Acetacetat, Aceton und Kreatinin - folgendermaßen mit alkalischem Pikrat zur Reaktion gebracht:

1) Ansatz nach Popper und Mitarbeitern (2)

In einem Zentrifugenglas werden $z u 1,0 \mathrm{~m} /$ Serum tropfenweise und unter Schütteln $3,0 \mathrm{ml}$ 1,2-proz. Pikrinsäure gegeben, der Niederschlag bei 5000 U./Min. $(3000 \mathrm{~g})$ abzentrifugiert, vom klaren Uberstand $3,0 \mathrm{~m} l$ in ein Reagenzglas abpipettiert und $0,15 \mathrm{ml}$ einer 10 -proz. $\mathrm{NaOH}$ zugesetzt. Die Ablesung der Extinktion erfolgt $15 \mathrm{Min}$. nach Zugabe der Lauge in einer 1-cm-Küvette gegen einen Leerwert mit einem Filterphotometer bei $546 \mathrm{~m} \mu$ (Hg-Lampe).

2) Ansatz nach BonsNes und TAUSSKY (3) $3,0 \mathrm{~m} l$ Serum werden in einem Zentrifugenröhrchen mit $3,0 \mathrm{ml}$ $\mathrm{H}_{2} \mathrm{O}$ bidest. verdünnt, $3,0 \mathrm{ml} 5$-proz. Natriumwolframatlösg. zugegeben und durch Zusatz von $3,0 \mathrm{ml} 0,66 \mathrm{~N}_{2} \mathrm{SO}_{4}$ das Eiweiß gefällt (nach Brod u. Sirota (4)). Nach $10 \mathrm{Min}$. Stehen wird der Fällungsansatz bei $5000 \mathrm{U}$./Min. $(3000 \mathrm{~g})$ zentrifugiert, vom klaren Uberstand $3,0 \mathrm{ml}$ in ein Reagenzglas abpipettiert und mit je $1,0 \mathrm{ml}$ einer $0,04 \mathrm{~N}$ Pikrinsäure und einer $0,75 \mathrm{~N} \mathrm{NaOH}$ versetzt. Ablesung der Extinktion wie beim Ansatz nach Popper.

Die verwendeten Chemikalien waren sämtlich p. a.-Präparate der Fa. Merck, Darmstadt. Das Acetacetat wurde nach einer Vorschrift von KREBS und EgGLeston (5) hergestellt; das Na-Salz der D(-)$\beta$-Hydroxybuttersäure wurde von der Fa. Serva, Heidelberg, bezogen. Die enzymatischen Bestimmungen erfolgten nach den Angaben von WILLIAMSON und Mitarbeitern (6) bzw. BERGMEYER und BERNT (7); das Enzym $\beta$-Hydroxybutyratdehydrogenase (HBDH) sowie die benötigten Coenzyme DPN und DPNH waren Handelspräparate der Fa. C. F. Boehringer, Mannheim.

\section{Ergebnisse}

Vergleich der Reaktionen von Kreatinin, Acetacetat und Aceton mit alkalischem Pikrat

\section{Absorptions-Spektren}

Bei der Reaktion von Kreatinin, Acetacetat und Aceton mit alkalischem Pikrat entsteht eine orange-rote Farbe, die mit bloßem Auge keine Unterschiede bei den 3 genannten Substanzen erkennen läßt. Nachdem von GarNer (8) das Absorptionsspektrum des Kreatininpikrats bereits beschrieben war, erschien es interessant, zum Vergleich auch die Spektren mit Acetacetat und Aceton aufzunehmen. In den Ansatz nach Popper wurden $0,15 \mu \mathrm{Mol}$ Kreatinin, 2,25 $\mu \mathrm{Mol}$ Acetacetat und $20,0 \mu \mathrm{Mol}$ Aceton in wäßriger Lösưng eingesetzt und jeweils gegen einen gleichzeitig alkalisierten Leerwert an einem registrierenden Spektralphotometer ${ }^{1}$ ) gemessen. Aus der Abbildung 1 ist zu erkennen, daß bei allen 3 Substanzen det entstandene Farbstoff sein Maximum zwischen 490 und $500 \mathrm{~m} \mu$ hat. Charakteristische Unterschiede im Kurvenverlauf sind nicht vorhanden, auch

1) Herrn Dr. H. Hetrier, Chem. Abteilung des MPI für experimentelle Medizin, Göttingen, schulde ich besonderen Dank für die Möglichkeit zur Messung an diesem Gerät. (Cary 14, Recording Spectrophotometer, Appl. Phys. Corp., Monrovia, Calif. USA.)

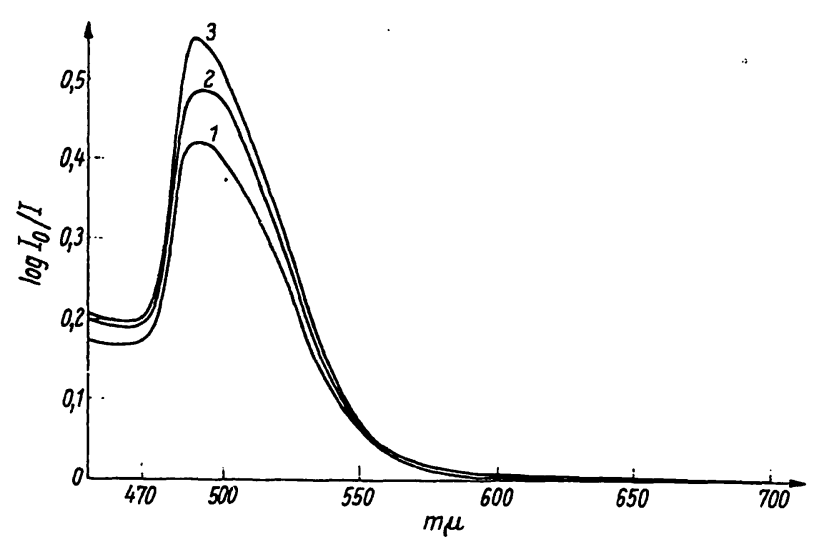

Abb. 1

Absorptionsspektren der mit Kreatinin (1), Aceton (2) und Acetacetat (3) in der JAFFE-Reaktion gebildeten Farbstoffe; Ansatz nach POPPER

die Zunahme der Extinktion verläuft mit etwa gleicher Geschwindigkeit und erreicht 15-20 Min. nach Zusatz der $\mathrm{NaOH}$ ihr Maximum.

\section{Molare Extinktionskoeffizienten}

Ließen sich bei der Darstellung der Absorptionsspektren zwar keine qualitativen Unterschiede zwischen Kreatinin, Acetacetat und Aceton feststellen, so deuteten doch die für eine etwa gleich große Farbbildung benötigten unterschiedlichen Konzentrationen der 3 Substanzen auf Differenzen quantitativer Art. Diese Unterschiede wurden in molaren Extinktionskoeffizienten bestimmt.

Wäßrige Lösungen von $0,1 \mathrm{M}$ Aceton, $0,0085 \mathrm{M}$ Acetacetat und $0,001 \mathrm{M}$ Kreatinin wurden in steigenden Mengen von 0,1 bis $1,0 \mathrm{~m} l$ in den Standardansatz nach Popper bzw. Bonsnes eingesetzt und aus dem Mittelwert der riach $15 \mathrm{Min}$. gemessenen, im linearen Bereich befindlichen Extinktionen der molare Extinktionskoeffizient berechnet. Der Gehalt der für diese Versuche verwendeten Acetacetat-Lösung wurde zum gleichen Zeitpunkt sowohl manometrisch nach Edson (9) als auch enzymatisch nach WILLIAMson (6) bestimmt)

$$
\text { Tab. } 1
$$

Molare Extinktionskoeffizienten bei $546 \mathrm{~m} \mu$ für Aceton, Acetacetat und Kreatinin in der Reaktion mit alkalischem Pikrat nach POPPER

\begin{tabular}{lrcrc}
\multicolumn{5}{c}{ und BONSNES } \\
\hline Substanz & POPPER & Quotient & BONSNES & Quotient \\
\hline Aceton & 9,8 & 1 & 1,8 & 1 \\
Acetacetat & 100,0 & 10 & 38,0 & 20 \\
Kreatinin & 1450,0 & 150 & 1740,0 & 1000 \\
\hline
\end{tabular}

In Tabelle 1 sind die Ergebnisse dieser Versuche und die in ganzen Zahlen ausgedrückten, auf Aceton bezogenen molaren Extinktionskoeffizienten zusammengefaßt. Die Werte für den BonsNes-Ansatz wurden unter Verwendung von Serum gewonnen. Die molaren Extinktionskoeffizienten des Acetons und Acetacetats sind wesentlich kleiner als der Extinktionskoeffizient des Kreatinins, besonders im Ansatz nach BonsNEs. Aceton wiederum liegt um einen Faktor 10 bzw. 20 niedriger als Acetacetat. Dies entspricht dem Verhalten in der Farbreaktion der beiden Ketonkörper mit Nitroprussidnatrium, die sämtlichen qualitativen Proben (LEGAL, LANGE, „Ketostix“, „Acetest") zum Nachweis von "Aceton im Urin" zugrunde liegt: auch dort ist das Auftreten der violetten Farbe bei Acetacetat um einen Faktor 10 empfindlicher, worauf bereits LORBER (10) hingewiesen hat. 


\section{Beseitigung des Störeffektes im Modellversuch}

Im Gegensatz zu einigen unbefriedigenden Versuchen mit Anilincitrat (9) gelang die Eliminierung der störenden Substanzen sehr einfach durch kurzes Kochen bei $100^{\circ}$, wobei Acetacetat unter $\mathrm{CO}_{2}$-Abspaltung in Aceton übergeht, welches wegen seines niederen Siedepunktes von $56,1^{\circ}$ flüchtig ist. Über die dabei nötigen Bedingungen gab folgender Versuch Aufschluß:

Von gepooltem „Normalserum" (,N"N) wurden $10,0 \mathrm{~m} / \mathrm{mit} 0,05 \mathrm{ml}$ einer $0,85 \mathrm{M}$ Acetacetatlösung versetzt; die Endkonzentration betrug also etwa $4 \mu \mathrm{Mol}$ Acetacetat $/ \mathrm{m} l$ Serum (,NA"). Je $6,0 \mathrm{~m} l$ $\mathrm{N}$-Serum und $6,0 \mathrm{~m} / \mathrm{NA}$-Serum wurden mit Wolframat enteiweißt, von den Uberständen $5 \times 3,0 \mathrm{ml}$ in Reagenzgläser abpipettiert, je 1 Probe als Ausgangswert beiseite gestellt, die restlichen Gläser in ein kochendes Wasserbad gesetzt und nach genau 1,2,5 und $10 \mathrm{Min}$. je 1 Reagenzglas der N-bzw. NA-Serie in ein Eisbad überführt. In je $0,05 \mathrm{~m} l$ der NA-Serie wurde Acetacetat enzymatischoptisch bestimmt, nit dem Rest die Farbreaktion nach BoNSNES durchgeführt. Die Ergebnisse dieses Versuches sind in Abbildung 2 wiedergegeben.

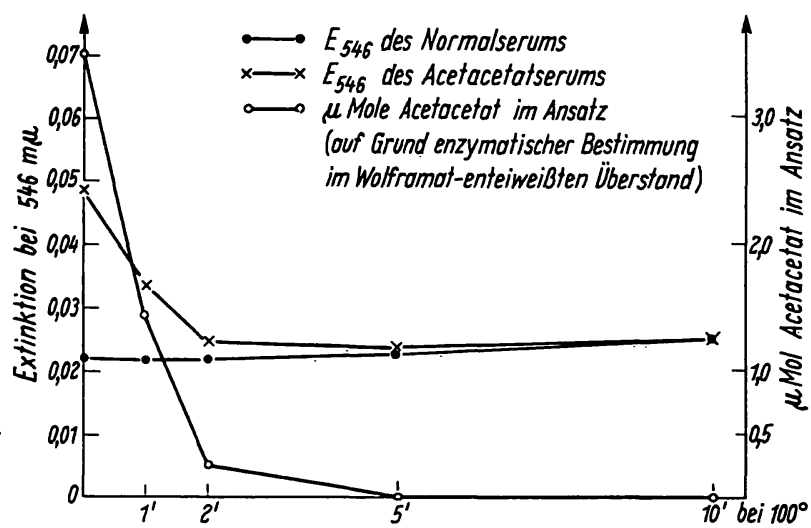

Abb. 2. Modellversuch zur Beseitigung des Ketonkörper-Störeffektes im Ansatz nach BonsNes
Der Versuch zeigt, daß sich durch Kochen bis zu 10 Min. die Extinktionen des Normalserums praktisch nicht verändern, während die durch das Vorhandensein von Acetacetat bedingte zusätzliche Extinktion im NASerum innerhalb von $5 \mathrm{Min}$. völlig beseitigt ist. Entsprechend geht das im enzymatischen Test bestimmte Acetacetat auf nicht mehr nachweisbare Mengen zurück. Die Anwesenheit von $\beta$-Hydroxybutyrat in einer Konzentration von $10 \mu \mathrm{Mol} / \mathrm{ml}$ Serum und von Glucose $(300,600$ und $900 \mathrm{mg} \%$ ) hatte keinen Einfluß auf die Beseitigung des Acetacetats in dem angegebenen Versuch.

\section{Diskussion}

Die erst kürzlich von Polar und Metcoff (11) sowie SLot (12) publizierten Arbeiten zeigen, daß es trotz vieler Bemühungen noch keine befriedigende $\mathrm{Be}$ stimmungsmethode für das Kreatinin im Serum gibt. Nach einer von Merten in den Jahren 1962 und 1965 durchgeführten Erhebung wird in den von ihm erfaßten Laboratorien das Kreatinin überwiegend nach der Vorschrift von Popper (2) bestimmt. Unter Berücksichtigung dieser Situation erscheint die in dieser Arbeit angegebene Methode einer Parallelbestimmung des Kreatinins vor und nach Beseitigung des Acetacetats und Acetons durch kurzes Erhitzen als die einfachste Möglichkeit, bei Ketonämie zu verläßlichen Kreatininwerten zu gelangen. Bei einigen bisher in unserer Klinik untersuchten Fällen stimmten die im Stadium der Ketoacidose ermittelten "wahren" Kreatininwerte sehr gut mit den nach Normalisierung der Ketonkörper erhaltenen Werten überein.

\title{
Literatur
}

1. JAFFE, M., Hoppe-Seyler's Z. physiol. Chem. 10, 391 (1886): 2. Popper, H., E.MAndel und H. Mayer, Biochem. Z. 291, 354 (1937). - 3. Bonsnes, R. W. und H. H. TAussky, J. biol. Chemistry 158, 581 (1945). - 4. Brod, J. und J. H. Sirota, J. Clin. Invest. 27, 645 (1948). - 5. KREBS, H. A. und L. V. EgGleston, Biochem. J. 39, 408 (1945). - 6. Williamson, D. H., J. Mellanby und H. A. KreBs, Biochem. J. 82, 90 (1962). - 7. BergmeYer, H. U. und
E. Bernt, Enzymol. Biol. Clin. 5, 65 (1965). - 8. Garner, R. J., Nature (London) 170, 460 (1952). - 9. Edson, N. L., Biochem. J. 29, 2082 (1935) zit. nach Manometric Techniques, UmbreitBurris-Stauffer, Burgess Publ. Minneapolis (1957). - 10. LORBER, L., Biochem. Z. 181, 375 (1927). - 11. PolaR, E. und J .MetcofF Clin. Chem. (New York) 11, 763 (1965). - 12. Stor, C., Scand. J. Clin. Laborat. Invest. 17, 381 (1965).

Dr. R. Kattermann, 34 Göttingen, Humboldtallee 1

\section{Akute Veränderungen der Serumproteine bei Ratten nach einmaliger Verabreichung von Barbital}

\author{
Von J. Neubaur \\ Aus dem Physiologisch-chemischen Institut der Universität Göttingen (Direktor: Prof. Dr. med. H.J. Deuticke)
}

(Eingegangen am 13. Juni 1966)

\begin{abstract}
Rattenserum wurde vor und nach Verabreichung einer einmaligen Dosis Bärbital im Stärkegel mit der Hochspannungselektrophorese getrennt. Danach erfolgte Proteinanalyse der Fraktionen mit dem Folin-Reagenz. Die slow- $\alpha_{2}$-Globulinfraktion war $6-36$ Stdn. nach Barbital-Verabreichung gegenüber den Werten vor Barbitalgabe und den Werten 1-3 Stdn. und denen 48-96 Stdn. nach Batrbital-Verabreichung erhöht. Die Erhöhung ist statistisch gesichert. Als Grund der genannten Veränderungen wird der Einfluß des Barbitals auf den Proteinstoffwechsel der Leber angesehen. Die Vorgänge scheinen für Barbital nicht spezifisch zu sein.
\end{abstract}

Rat serum was fractionated by high voltage electrophoresis in starch gel before and after the administration of a single dose of Barbital. The fractions were then analysed for protein with Folin's reagent. The slow- $\alpha_{2}-$ globulin fraction was increased 6-36 hours after the administration of Barbital, compared with the values before Barbital and with the values 1-3 and 48-96 hours after Barbital. The increase was statistically significant. The changes are probably caused by an effect of Barbital on protein metabolism in the liver. The reactions do not appear to be specific for Barbital. 